\title{
Design of Office Seats for Meeting Room Environment and Visitors Area Based on Ergonomics
}

\author{
Liu Jinghui \\ HHUC 200 Jinling North Road, Changzhou, Jiangsu Province
}

\begin{abstract}
The design functions of office seats in the market are relatively weak. According to the requirements of different sitting positions in the office environment and the physiological and morphological characteristics of sitting positions, this paper analyzes the seats from the perspective of ergonomics to design conference room seats that are more suitable for forward leaning sitting positions. The research method adopts the investigation method, literature research method and other investigation methods to find the existing problems of office seats at present, and analyzes and improves the design in combination with relevant literature. The office seats in small conference rooms and visitor areas are redesigned to obtain office seats that are easy to move and conform to the humancomputer interaction of the sitting posture of leaning forward in meetings.
\end{abstract}

\section{$1 \quad$ Introduction}

Under the background of modern society, the contact time between indoor workers and office chairs occupies most of the working time of the day. At present, most seats are designed based on the static size of the human body, while ignoring the user needs and dynamic health under the specific seat use environment. In reception areas and small conference rooms, office seats are no longer used by individuals. Users only use the seats for a short period of time and often do not adjust the specific seat height and armrest position of the seats. Instead, they often move the seats, which reduces the comfort level of the seats and ignores many other functions. The design of office seats needs to be integrated with sitting posture in different office scenes to help users better optimize their office experience [1].In diverse use conditions such as meetings, people's sitting posture is often transient and keeps a state of leaning forward posture. Users may also carry notebooks, briefcases and other articles according to meeting requirements, which are inconvenient to place. In response to such phenomena, we will combine the actual product requirements with ergonomics, improve the adjustment and design of the seat structure, design the meeting room seat that is suitable for the reclining sitting position, add functions such as storage and easy movement, and improve work efficiency and comfort.

\section{Design of ergonomics Seat Based on Dynamic Health}

Seat functions are essential furniture in people's daily life, including communication, rest, office, which need seats to support the body. In the office environment, people use the seat for a long time, so the comfort of the seat is particularly important for health. In addition, the chair should also provide good human-computer interaction experience to improve office efficiency. According to scientific research, a healthy posture needs to be dynamic, based on frequent changes in sitting posture. Therefore, the design of seat dimensions should take full account of the static dimensions of the human body and take the interaction between people and seats as the consideration point [2].When designing a seat, the design of the seat should be considered comprehensively from the static size and dynamic size of the human body. The static size of human body mainly refers to the basic physiological scale of human body, while the dynamic size refers to the change of scale under the influence of activity factors in the process of activities and furniture use. Dynamic factors require that when designing furniture seats, the common postures in use and the dimensional changes caused by them should be fully investigated. The seat should also have certain adjustable functions according to dynamic factors to meet the interactive requirements.

Static size includes seat depth and basic size of high chair. Static size is the basis of seat design, which determines the shape and basic functions of the seat. In the relevant design, we should consider the user's body size to meet the user's basic product needs.

The dynamic size should be established during the user's use. People usually push and pull the chair to adjust the chair position or change the steering direction and adjust the backrest etc. Each action frequency is different under different usage scenarios. When considering dynamic size, we should refine the user base in order to accurately refine the user's needs and calculate the seat dynamic size. The aim is to promote a seating 
behavior in which form, function and ergonomics work together in an almost symbiotic manner. Considering the design comprehensively, adding dynamic adjustment factors can greatly improve the seat comfort and optimize the human-computer interaction experience, thus improving office efficiency or improving human health.

\section{Dynamic Size Analysis of Seats in Meeting Rooms and Visitors' Area}

\subsection{Design Defects of Office Seats in Meeting Room Environment or Visitors' Area}

Most chairs only consider the static size of the human body. Under the use environment such as meeting rooms or the visitor area, users push and pull chairs, turn according to communication needs, record or work at desk, and get up frequently without excessively adjusting the height angle of the chairs according to their own needs. In view of the problems in the process of humancomputer interaction, we found that the current office chair does not have a handle so it is inconvenient to move by design. And the noise is loud when moving due to weight problems. We need keep the environment quiet when working in the conference room or the visitor area, and move the seats with quiet and easy-to-use handrails. Most armrests are not adjustable and their shape is not suitable. Long-term use will damage the wrist and the arm will be in an unnatural bent state during rest. Most users need to use a mouse and the like during meetings, which increases wrist burden, does not have suitable wrist support parts, and long-term ulnar deviation or wrist rotation may lead to tenosynovitis , which reduces work efficiency. Most seats have no other arrangement except armrests on the side and have no storage design, so it is not convenient to place personal belongings. Articles are easy to slide off the side or back, which may cause interference in the office. There is no rotation design for the seat, so it is not convenient to adjust the angle according to the needs during the meeting. Most seats are equipped with chair wheels, but the center of gravity is not stable enough, which may lead to misoperation and noise when sliding. For common sitting postures, the stress on the feet is not taken into account, which may cause problems such as poor blood circulation to the thighs and inconvenience in getting up, which reduces work efficiency.

\subsection{Optimize the design and human-computer interaction}

Optimizing the human-computer interaction experience requires us to refine the user's high-frequency actions in a specific environment, establish the corresponding relationship between actions and a certain requirement of the seat for consideration and optimization, and add dynamic adjustment factors. The meeting room should be kept quiet, so there are handles designed to move easily in the seat, and the weight is small. According to the specific usage scenario, the headrest and shoulder rest should be removed to reduce the weight of the seat. As a small conference chair, it should have a rotation function to facilitate the adjustment of the orientation according to the needs of the council, without the need to frequently slide the seat which makes noise. The user may use a notebook or the like while in the conference room, and the wrist burden is heavy, so it is necessary to pay attention to the rest of the wrist and elbow. Therefore, the seat needs to be provided with an adjustable armrest, and the wrist arm is in a relaxed state when resting. The feet should be fully in contact with the ground to ensure the strength and comfort of the seat. When the seat rotates, consider the appropriate angle of the feet and legs to facilitate sliding. As participants usually carry paper and pens, briefcases, notebook computers or other items, the design points include: paper and pen placement, briefcase hook, mouse operation table. The seat should have a storage design, a small item storage at the armrest and a briefcase hook to facilitate placing articles and they are integrally arranged at the armrest position. After adding the briefcase suspension design, the mobility of the seat will be reduced, so it is decided to focus on the improvement of the seat design on the stability of the seat and cancel the movement function of the seat. According to the sitting habits of the meeting, we consider the design that the seat can slide forward as the user leans forward. Due to the fact that in the meeting room or the visitor area, users are mostly in a forward leaning sitting position for listening, the seat depth is appropriately reduced to reduce the movement of the center of gravity. After establishing the correspondence between usage actions and user needs, we carry out targeted improvement and optimization in the design, comprehensively considering dynamic factors and static dimensions.

\section{$4 \quad$ Design Elements of Meeting Room Seats and Visitors' Area}

After refining the correspondence between the highfrequency actions of the users in the conference room or visitor area and the users' requirements, the dynamic size is considered based on the static size of the human body and dynamic adjustment factors are added to the seats. The user's needs are mainly reflected in four aspects: convenience for desk work, convenience for moving the seat, convenience for communication and listening, and convenience for placing articles. The corresponding requirements are reflected in the seat design, which needs to consider the convenience of work design and improve work efficiency, so as to meet the requirement that the seat can be moved in silence, and steering and leaning forward can be carried out according to the user's independent steering and forward leaning without too much self-adjusting adjustment device. We focus on the armrest, movement, steering and storage of seats. 


\subsection{Armrest Design}

The armrest is a component that provides support for the elbow, to a certain extent, it can relieve the fatigue of the arm and wrist to prevent the occurrence of elbow and wrist fatigue syndrome. Long-term ulnar deviation of hand or wrist rotation may cause tenosynovitis and other hand diseases. Most people will use the mouse and so on during meeting, which increase the burden on their hands. Based on the dynamic size adjustment, the right armrest is provided with an adjustable angle structure which can place a mouse, etc. to support the wrist. Taking into account the size that most people can fit, the 90th percentile $180 \mathrm{~mm}$ shown in Chart 1 is adopted, so that

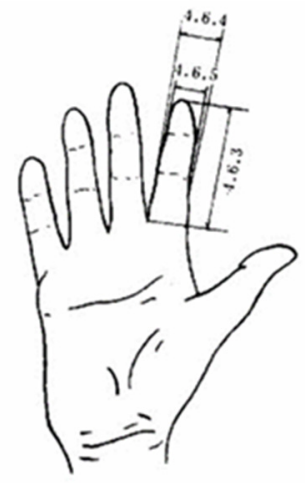

most people's hands can be evenly placed on the armrest. The mouse can be freely moved while the hands naturally bend and grasp the mouse, and the mouse can be conveniently moved and operated by setting the same width. Some trivial things can also be placed on the platform. The supporting part can be turned up and down and adjusted by 15 degrees, so that the wrist is in a naturally relaxed state. The distance between armrests should be slightly wider than the horizontal dimension of human body, and should match the width of chair back and seat surface. Thus, the height of the handrail is about $200 \mathrm{~mm}$ from the seating surface, the length is $200 \mathrm{~mm}$ to $250 \mathrm{~mm}$, and the width is $40 \mathrm{~mm}$ to $60 \mathrm{~mm}$.

\begin{tabular}{|l|llllll|}
\hline \multicolumn{7}{|c|}{$18-55$} \\
\hline Measurement & 1 & 5 & 10 & 50 & 90 & 95 \\
\hline 4.6.1 Hand length & 154 & 159 & 161 & 171 & 183 & 189 \\
\hline 4.6 .2 Hand width & 67 & 70 & 71 & 76 & 82 & 84 \\
\hline $\begin{array}{l}\text { 4.6.3 Index } \\
\text { Finger length }\end{array}$ & 57 & 60 & 61 & 66 & 72 & 76 \\
\hline
\end{tabular}

Chart 1 Static Dimensions of Human Hand

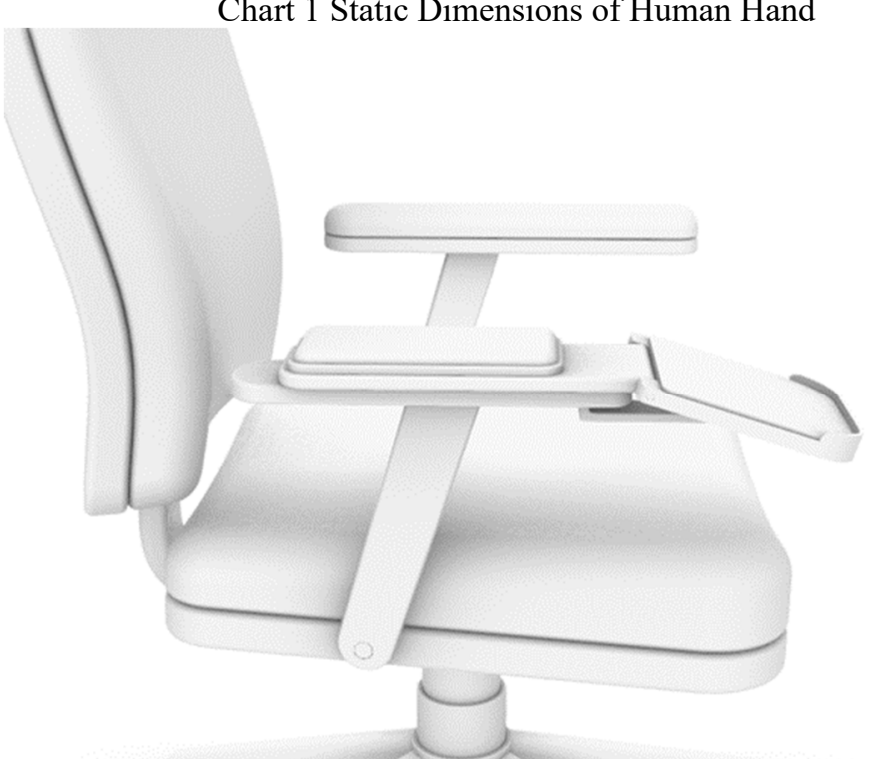

Chart 2 Armrest Design Effect Diagram

\subsection{Portable Design}

Frequent chair movement is required in the meeting room or visitor area. Most of the seats have no handles that are easy to move. In order to facilitate the quiet movement of the seat and meet the use requirements, the side of the seat and the bottom of the seat are both provided with recesses for easy handling. When sitting, the bottom of the seat also has recesses that are easy to adjust the seat. The low backrest is designed to reduce weight,. Considering ergonomics, we want to be able to move the seat from a standing position without bending the elbow or waist too much. So we took the 50th percentile on both sides of the seat and made a recess in the $741 \mathrm{~mm}$ height ,allowing most people to move the seat without bending their elbows too much. 


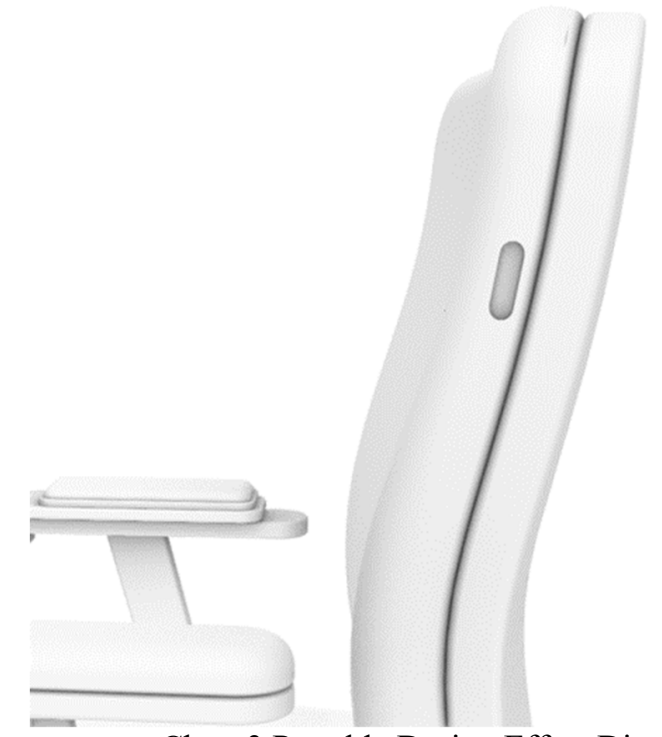

Chart 3 Portable Design Effect Diagram

\subsection{Steering and Adjustable Forward Lean Design}

In the conference room and the visitor area, users need to change the orientation and lean forward at any time to facilitate listening and communication. In this paper, we use no wheelchair design. Because of no wheelchair, the seat cannot slide, but can be quietly rotating, convenient for users to adjust according to their needs. The seat cushion can slide forward with the user leaning forward to facilitate communication, so that the seat is more suitable for meeting room requirements and has the functions of stabilizing the center of gravity and quiet steering. When rotating the seat, the feet should fully contact the ground to ensure comfort of the seat. Therefore, when rotating, the distance between the thigh and the calf should be slightly less than $80^{\circ}$, and the footstep should be kept horizontal to facilitate force support. At the same time, considering the forward leaning posture, we adopt a seat depth of $380 \mathrm{~mm}$ so that the center of gravity will not move greatly when leaning forward, the feet can fully contact the ground, keep horizontal with the ground and the angle between thighs and calves is slightly less than $90^{\circ}$, which makes the seat more stable and the sitting posture more comfortable. The cushion can move smoothly by $25 \mathrm{~mm}$ as the user leans forward, facilitating communication and listening.

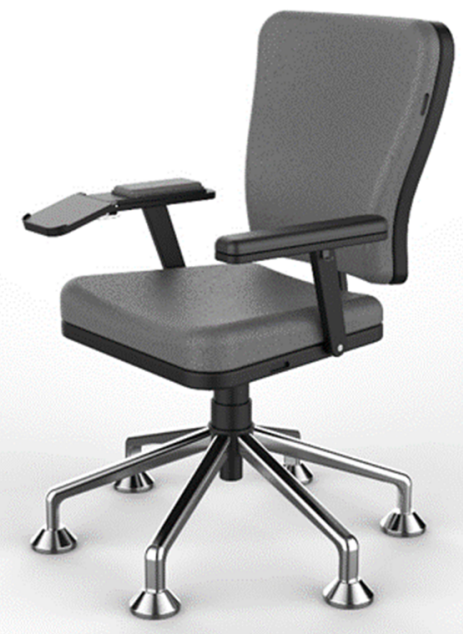

Chart 4 Effect Diagram of Rotation and Forward Tilt Design

dynamic adjustment, only static size analysis can meet the needs. A hook is arranged on the side of the armrest

\subsection{Design of Storage Hook}

There is limited space in the visitor area or conference room. Participants or visitors will carry notebooks or bags and other items according to their needs and there is no place to put them. This design does not involve user meeting the requirement of human-computer interaction and is used for hanging briefcase and the like, so that a user can conveniently take and place articles from the side even in a sitting posture. 


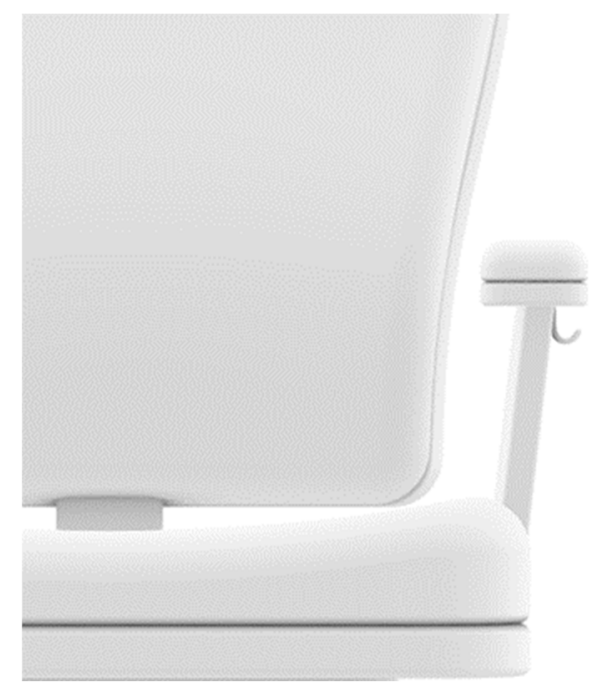

Chart 5 Hook Design Effect Diagram

\subsection{Other Dimension Design}

In a sitting posture, the body is supported mainly by the spine, pelvis, legs and pedals. Normal column curvature are the keys to sitting comfortably 3 . In the specific use environment of the conference room or visitor area, the seats are generally non-personalized seats, which can be used within a short period of time without the need for extra specific adjustable size. People generally lean forward, and most of the time they do not need to use shoulder rest and headrest for rest. Therefore, a suitable lumbar design is sufficient to meet the design requirements of the visitor area seats. The design of the lumbar support should meet the requirements of the human sitting posture, and it is a component that has the function of alleviating waist fatigue caused by prolonged sitting. Generally, the lumbar support is taken as $\mathrm{g}=165$ $\sim 210 \mathrm{~mm}$. According to the design parameters of the work seat of Chinese adult human body and scholars such as Tian Liying [3] the ergonomic seat size that can be used by most people can be obtained. The seat width of the work chair is $400 \mathrm{~mm}$ and the waist is $330 \mathrm{~mm}$ It makes the waist support in sitting position more comfortable. Seat height is usually $350-507 \mathrm{~mm} \mathrm{4}$. Since the spine of the human body is naturally bent when seated, the back of the chair should have a certain inclination angle, and at the same time, the part of the backrest should have a certain supporting effect [5], in the case of the two supporting points of the cushion and the lumbar pad, the 115 inclination angle of the lumbar lean is more suitable for the curve of the spine of the sitting posture.

\section{$5 \quad$ Conclusion}

People pay more and more attention to health, so the combination of dynamic adjustment factors and static size for furniture design may become the key point of design practice. Starting from the seat design in the use environment of the visitor area and small conference room, this paper studies the main sitting posture and corresponding physiological characteristics of the human body in the visitor area and conference room, and designs them in combination with dynamic and static dimensions. In the meeting room scene, when people sit and listen or work, they are leaning forward most of the time. At this time, it is mainly supported by the seat cushion and waist cushion, while the headrest and shoulder rest have less effect. On this basis, considering the user's expected needs, participants or visitors may carry briefcases and other items with them, and considering the storage design, the user should reduce the wrist burden during sit, be easy to operate, and design wrist adjustment rest devices. In the use environment of the conference room, the chair with no chair wheel rotation design is adopted. This paper focuses on the design concept, combining dynamic and static dimensions, and discusses the design of office seats suitable for meeting rooms and visitors areas. Explore and optimize the design of human-computer interaction experience under different specific use environments. Due to changes in national standards, there may be some deviations in the dimensions or data involved in this paper.

\section{References}

1. Sun Xinxin, Xu Baiming, SunXinxin, et al. Research on Office Furniture Design Based on Health Concept $[\mathrm{J}]$. Furniture and Interior Decoration, 2017(11)..

2. Zhang Mi. Research on Application of Ergonomics in Furniture Design [J]. Intelligent City, 2017, 3(01): 311 .

3. Tian liying. design and research of office desks and chairs based on ergonomics [D]. Henan university of science and technology.

4. Lu Jianxiong, Zhang Fuchang, Shen Limin. Ergonomic Discussion on Sitting Position and Seat Design [J]. Jiangnan University, 2005(4):45-46.

5. Sun Minglei, Ha Jianing, Gao Hongbo. Research on Scale Design of Seat Furniture Based on Human Dynamic Factors [J]. Art and Technology, 2019(7):184-184. 